\title{
Reliability Analysis of Railway Signaling System Based Petri Net
}

\author{
Hongxia Chen ${ }^{\text {a }}$, Qiang Sun \\ Nanjing Institute Railway Technology, Nanjing 210031, China \\ achen.hongxia9@gmail.com
}

Keywords: Railway signaling; Fiber-optic communication; Petri net; Markov process

Abstract. Railway signaling system is essential equipment for high speed railway and passenger special line, and it play an important role to ensure safety and improve efficiency. Reliability analysis to railway signaling system is necessary and valuable. Railway signaling system is a complex and dynamic system. Petri net is a powerful mathematic tool to deal with concurrent and dynamic system. Taking hot standby system as an example, this paper uses petri net to analyze hot standby system and establish system modeling under the condition of using fiber-optic. Markov process and stochastic process are also used to compute the system reliability. The analysis process is accord to the actual working condition. It has important reference value to the other system design and analysis.

\section{Introduction}

With the development of high speed railway and passenger special line, railway signaling systems have become one of the four core technology of high speed railway. Railway has speeded up for six times. In order to ensure railway departments' coordination, railway transport high-speed, safety and accuracy, railway signaling systems have become the brain of railway transport, and reliability also gives railway signaling system sacred mission. Previously, the speed of trains was low, railway communication system and signal system basically is independent. Today, Railway system tends to comprehensive. This separation condition does not adapt with the development needs, because much information which involves train dispatching, controlling, monitoring of signal systems need conveying through the communication system. Communication and signaling have combined and formed the integrated signaling systems.

The reliability of the integrated railway signaling system is decided by two sides which are the hardware reliability and software reliability. This paper mainly analyzes the hardware reliability. With host and standby as the example, the system uses $1+1$ hot backup and switching device has been set between the host and standby. Transmission channels also use redundancy way to ensure the reliability of the information transmission. These several factors to affect the signaling system are dynamic process in ensuring system reliability. This paper uses petri net to analyze the signaling system. Petri net is the most effective analysis method to dynamic concurrent systems [1,3]. This signaling system also uses optical communication system as transmission channel; the optical communication system makes the main optical fiber and standby fiber in different cable of the unified routing. This paper establishes the system modeling and reliability modeling. Finally this paper analyzes the system reliability.

\section{System Modeling}

The concept of the petri net has its origin in Carl Adam petri's dissertation, submitted in 1962. It is a promising tool for describing and studying information processing systems that is characterized as being concurrent, asynchronous[2,4]. It also describes discrete dynamic system which is isomorphic to continuous time Markov chain. These characteristics can be easily used for modeling of discrete dynamic system, performance and reliability analysis. So petri net is used for railway signaling system modeling based on optical fiber communication system. Fig. 1 is the system model. 


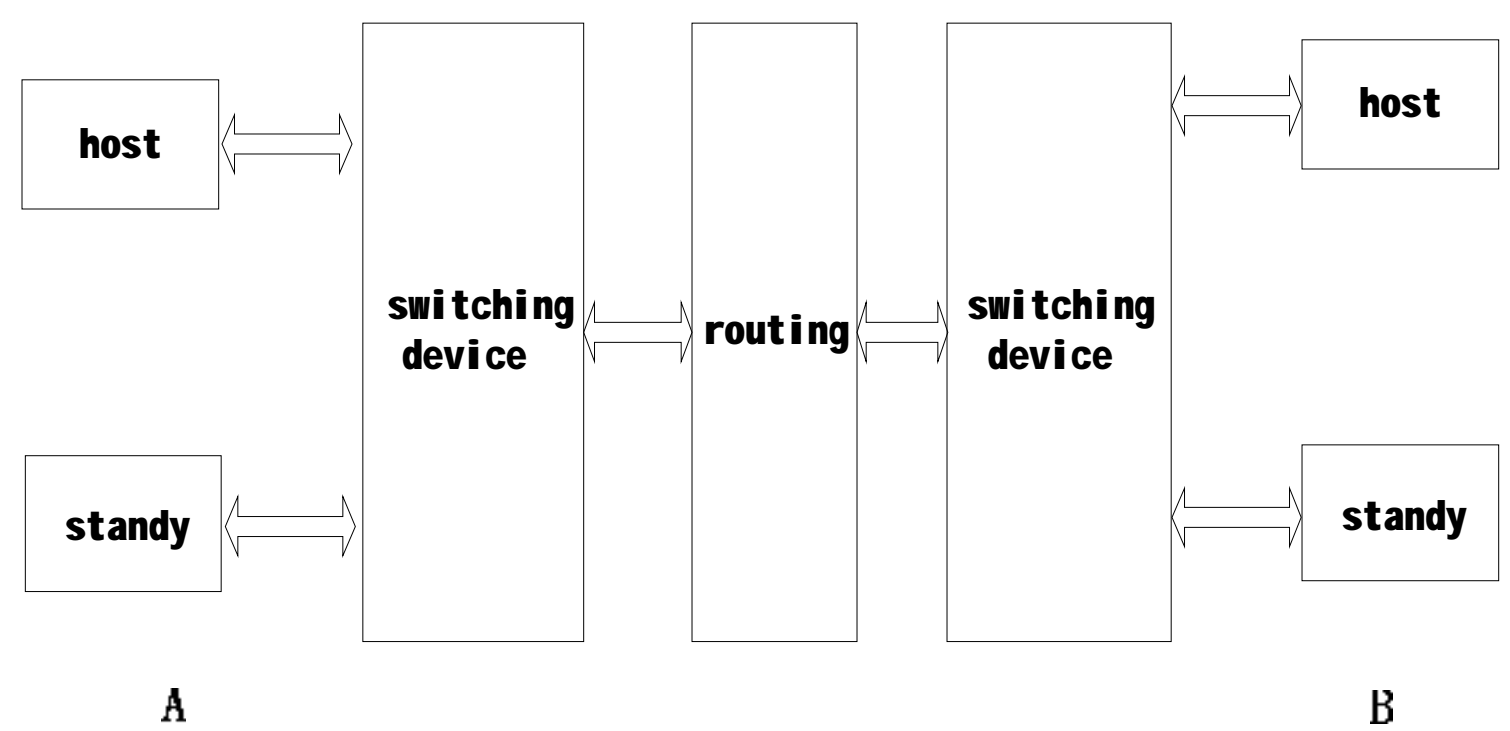

Fig. 1 system model

As illustrated in Fig. 1, this signaling system is composed of host, standby, switching device and routing equipment. Fig. 1 shows that the system can be divided into two subsystems: signal devices subsystem and routing equipment subsystem. The whole system reliability is decided by signal equipment subsystem reliability and routing device subsystems. The whole system reliability is product of the two subsystems which are series system.

When part of the signaling system is in trouble, the maintenance staff should repair fault parts quickly. Usually different maintenance ways have been used in the repair process according to the type of equipment. Usually to electricity units, routine maintenance such as replacing the fault equipment need to check the relevant units, but if the fault affects the normal operation, maintenance personnel must make an emergent action to replace the whole component even that relevant units is good[5].

\section{System Reliability Modeling}

Signal Devices Subsystem Modeling. Signal devices subsystem is the dual module hot spare. Host and the standby are in simultaneous working state and have special failure rate. When switching device normally, if host is in fault, standby will prepare for a chance to upgrade. Usually switching time is very short in hot backup condition. This paper assumes the conversion time is zero. Fig. 2 is the dual module hot spare signal equipment subsystem model. The subsystem is repairable.

The meaning of symbols in Fig.2 as follows.

$p_{1}:$ Host operating normally;

$p_{2}:$ Switching device operating normally ;

$p_{3}:$ Standby operating normally;

$p_{4}$ :Host failure for repairs;

$p_{5}:$ Switching device failure for repairs;

$p_{6}:$ Standby failure for repairs;

$p_{7}$ :Signal equipment failure state because of host and switching device in fault;

$p_{8}$ : Signal equipment failure state because of host and standby failure, marked failure occurred;

$t_{1}$ :Simulate host failure process;

$t_{2}$ :Simulate switching device failure process;

$t_{3}$ :Simulate Standby failure process; 
$t_{4}$ :Signal equipment failure because of host and switching device fault;

$t_{5}$ : Simulate switching units for repair process in devices good-state cases;

$t_{6}:$ Signal equipment failure because of host and standby fault;

$t_{7}$ : Simulate standby for repair process in devices good-state cases;

$t_{8}$ : Simulate fault unit for urgent repair process in $p_{7}$ state;

$t_{9}$ : Simulate fault unit for urgent repair process in $p_{8}$ state;

$t_{10}$ : Simulate switching of host and standby in host fail-state, standby and switching device good-state;

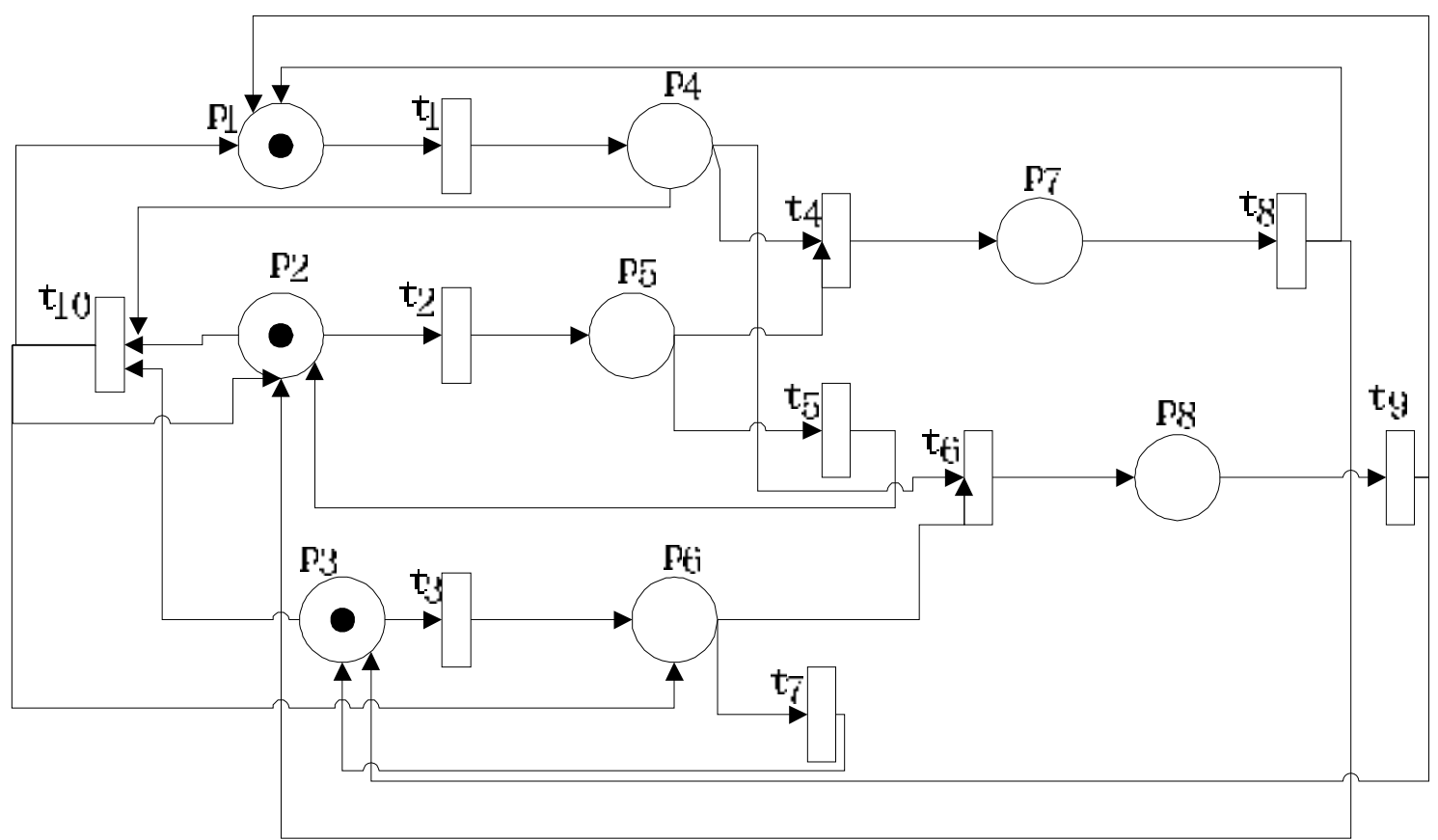

Fig.2 Signal equipment subsystem petri net model

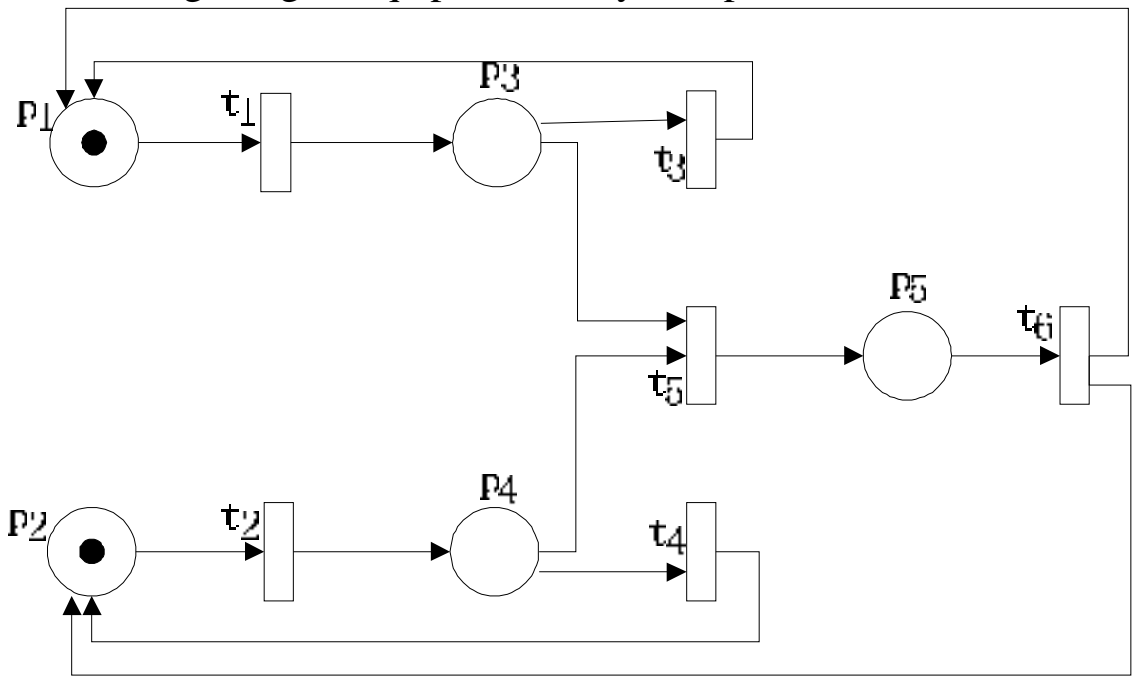

Fig.3 Routing equipment subsystem model

Routing Equipment Subsystem Modeling. Routing equipment subsystem utilizes the redundant technology, namely in parallel. Routing equipment subsystem will fail only routing equipment all in fault. Signaling system demands that redundant units cannot affect the whole systems except downgrading, so maintenance personnel must carry out emergent repairing. Fig.3 shows routing equipment subsystem model. 
The meaning of symbols in figure 3 as follows.

$p_{1}$ : Routing system A working normally marked;

$p_{2}$ : Routing system B working normally marked;

$p_{3}$ : Routing system A in failure;

$p_{4}$ : Routing system B in failure;

$p_{5}$ : Routing system in failure;

$t_{1}$ : Simulate routing system A failure process;

$t_{2}$ : Simulate routing system B failure process;

$t_{3}$ : Routing system A failure for repairs;

$t_{4}$ : Routing system B failure for repairs;

$t_{5}$ : Simulate fault unit for urgent repair process.

\section{System Reliability Analysis and Calculation}

In order to know the process of operating and switching of the system, reachable tree which is one of the important tools for Petri net to analyze working process of the system is used. The reachable tree can be drawn as in Fig.4. Here the initial state is $\mu_{0}=\left(p_{1}, p_{2}, p_{3}, p_{4}, p_{5}, p_{6}, p_{7}, p_{8}\right)$, and then $\mu_{0}=(11100000)$ is the initial marking in Fig. 4.

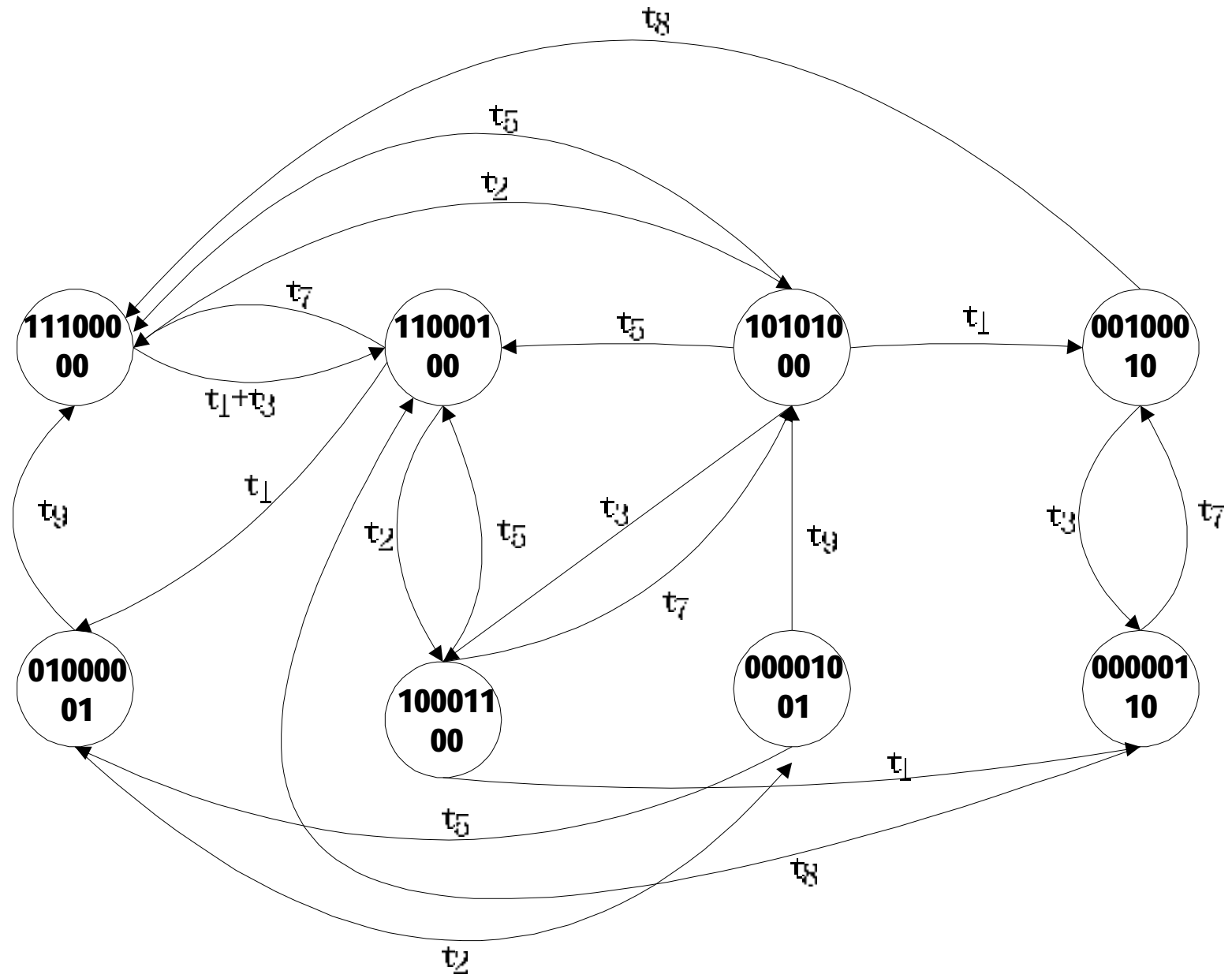

Fig.4 contracted reachable tree of modeling in Fig.2 showed 
Generally, the average electronic equipments obey index distribution, so here assuming: the failure ratio of host and standby $\lambda_{1}=0.001$, the failure ratio of Switching device $\lambda_{2}=0.0005$, equipment routine maintenance ratio $\mu_{1}=0.01$, urgent repairing ratio $\mu_{2}=0.05$.

$$
\sum_{j=1}^{n} p_{j i}=1, i=1,2, \Lambda, n
$$

According to stochastic process and Markov process, the stable state probabilistic distribution $\mathrm{Y}$ can be concluded by formula (1), namely $\mathrm{Y}=(0.807360 .14525,0.03833,0.00528,0.00289,0.00076$, $0.00007,0.00006)$. Y value is compute by software Matlab. When equipment is available, according to the reliability calculation method of series system, reliability is 0.99622 .

Reliability of routing equipment subsystem can be obtained in the same method. We can get the system reliability is 0.999902 assuming the unit failure ratio $\lambda=0.001$, maintenance ratio $\mu=0.1$.

The signaling system reliability is the product of signal equipment reliability and routing equipment reliability, namely 0.995902 .

\section{Conclusion}

This paper mainly analyzes railway signaling system reliability under the condition of using fiber-optic. It uses petri net to analyze and establish modeling, and then it computes the reliability of system using Markov process. This signaling system analyzed is accord with the actual working process. It proved the feasibility of the methods, and it shows the advantage of Petri nets to analyze dynamic system and have references to system design analysis.

\section{References}

[1] YuanChongYi,Petri nets principle and application,Beijing,Electronic Industry Press,2005,p.32-100.

[2] LiDongQi, ma jian fan-yun yu. A high usability redundancy cluster analysis of the key technology and credibility. Chengdu, Information Engineering College Journal,Vol.24(3),p.245.

[3] HuangXueMei, HuJianSheng etc. optical fiber communication system available performance analysis Based on generalized stochastic petri nets,Science and Technology Information,No.7,p.66-67,2009.

[4] S.Fararooy and J.Allan. Condition-based Maintenance of Railway Signalling Equipment, Electric Railways in United Europe, 1995, International Conference.

[5] Jin Guo,Hongxia Chen, and Changqian Zhu, Design of micro-computerized dual-computer redundant automatic block system between railway stations, Journal of Southwest Jiaotong University, (2001)Vol.40, p484-488. 